\title{
ALTERAÇÕES CLÍNICAS E LABORATORIAIS COMO INDICADORES PARA O TRATAMENTO ANTI-HELMÍNTICO EM OVINOS EXPERIMENTALMENTE INFECTADOS COM Haemonchus contortus
}

\author{
CLINICAL AND LABORATORIAL ALTERATIONS AS INDICATORS FOR \\ ANTI-HELMINTIC TREATMENT IN SHEEP EXPERIMENTALLY INFECTED \\ WITH Haemonchus contortus
}

\author{
Bárbara Nascimento Lemos Hupp ${ }^{1}$ \\ Marcelle Temporim Novaes ${ }^{1}$ \\ Marcela Santos Sena Martins ${ }^{1}$ \\ Adriano Conti Hupp ${ }^{1}$ \\ Leonardo Oliveira Trivilin ${ }^{1}$ \\ Isabella Vilhena Freire Martins ${ }^{1 *}$
}

1 Universidade Federal do Espírito Santo, Alegre, ES, Brasil.

*Autor para correspondência - ivfmartins@gmail.com

\begin{abstract}
Resumo
A intensidade da anemia e hipoproteinemia são indicadores da gravidade da hemoncose. Este estudo objetivou verificar as alterações de peso, hematócrito e proteína plasmática, bem como a carga parasitária que indiquem a necessidade de intervenção anti-helmíntica em ovinos experimentalmente infectados com Haemonchus contortus. Foram utilizados 14 ovinos machos mestiços Santa Inês, 42,5 $( \pm 7,5) \mathrm{Kg}$, livres de nematoides, divididos em dois grupos contendo sete animais cada. Os animais do grupo infectado receberam 10.000 larvas de $H$. contortus cada. Todos os animais foram acompanhados aos 21, 28, 35, 42, 49, 56, 63 e 70 dias pós-infecção e avaliados segundo os parâmetros: OPG, Famacha ${ }^{\odot}$, peso, proteínas plasmáticas totais e hematócrito. No grupo infectado, o OPG variou e atingiu seu ápice aos 42 dias de acompanhamento, o hematócrito foi significativamente menor a partir da infecção experimental, o escore Famacha ${ }^{\odot}$ variou de 1 a 2 e significativamente maior aos 21, 28, 35 e 42 dias, não houve alteração no nível de proteína plasmática nem no peso corpóreo em relação ao grupo controle. A infecção com até 10.000 larvas de H. contortus e OPG até 2500 não afeta significativamente o peso, hematócrito e proteína plasmática de ovinos mestiços Santa Inês, não havendo necessidade de intervenção anti-helmíntica.
\end{abstract}

Palavras-chave: carga parasitária; controle; pequenos ruminantes.

\begin{abstract}
The intensity of anemia and hypoproteinemia are indicators of the seriousness of haemonchosis. The objective of this study was to verify the changes in weight, hematocrit, and plasma protein, as well as the parasite load, indicating the need for anthelmintic intervention in sheep experimentally infected
\end{abstract}


with Haemonchus contortus. Fourteen crossbred Santa Inês male sheep were used, weighing 42.5 ( \pm $7.5) \mathrm{kg}$, nematodes-free. The animals were divided into two groups of seven animals each. The group of infected animals received 10,000 $\mathrm{H}$. contortus larvae each. All animals were monitored after 21, $28,35,42,49,56,63$, and 70 days post-infection and the following parameters were assessed: EPG, Famacha $^{\odot}$, weight, total plasma proteins, and hematocrit. In the infected group, the EPG varied and peaked on the 42nd follow-up day. The hematocrit became significantly lower after the experimental infection. Famacha score ${ }^{\odot}$ ranged from 1 to 2 and it was significantly higher at 21, 28, 35 and 42 days. No change was observed in plasma protein level and body weight in the control group. Infection with up to 10,000 larvae of $H$. contortus. and EPG up to 2500 did not significantly affect weight, hematocrit, and plasma protein of crossbred Santa Inês sheep with no need for anthelmintic intervention.

Keywords: control; parasitic load; small ruminants.

Recebido em: 24 de agosto de 2016

Aceito em: 11 de dezembro de 2017

\section{Introdução}

A ovinocultura vem ganhando destaque no agronegócio brasileiro a cada ano e surge como uma alternativa econômica para pequenos e médios produtores ${ }^{(1)}$. Um dos pontos mais importantes relacionados à ovinocultura, além dos fatores nutricionais, é a verminose, que representa um dos maiores desafios na produção de carne ovina ${ }^{(2)}$.

A patogenia da verminose e o aparecimento de sinais clínicos podem variar de acordo com a idade do hospedeiro, a imunidade adquirida em infecções prévias, o estado nutricional, a intensidade da carga parasitária e as espécies de nematoides envolvidos ${ }^{(2)}$. Assim, a presença do parasito não significa necessariamente a ocorrência da doença, uma vez que o aumento da resistência desenvolvida pelo animal pode limitar o desenvolvimento ou mesmo levar à eliminação dos parasitos presentes ${ }^{(4-}$ 6).

Em pequenos ruminantes, a intensidade da anemia e da hipoproteinemia são usualmente os indicadores da gravidade das parasitoses gastrintestinais, principalmente as ocasionadas pelo parasito Haemonchus contortus $^{(7)}$. Por isso, estudos que associam alterações hematológicas, como do volume globular, à contagem de ovos por grama de fezes (OPG) podem auxiliar no diagnóstico e na seleção de animais resistentes ${ }^{(8)}$.

Este estudo objetivou avaliar possíveis alterações nos parâmetros peso, hematócrito, proteínas plasmáticas totais, ovos por grama de fezes e método Famacha como indicativo da necessidade de intervenção anti-helmíntica em ovinos experimentalmente infectados com $H$. contortus.

\section{Material e métodos}

Os animais utilizados neste estudo foram mantidos de acordo com a Lei Brasileira de Procedimentos para Uso 
Científico de Animais ( $\left.\mathrm{N}^{\circ} .11794 / 2008\right)$. Os procedimentos experimentais foram revisados e aprovados pelo Comitê de Ética e Utilização de Animais da Universidade Federal do Espírito Santo de acordo com o protocolo 015/2013.

O experimento foi conduzido no Setor de Ovinocultura do Instituto Federal de Educação, Ciência e Tecnologia (IFES), Campus de Alegre, situado no distrito de Rive/Alegre-ES, coordenadas (204' $30^{\prime \prime}$ Sul, $41^{\circ} 27^{\prime} 23^{\prime}$ ' Oeste e altitude de 138 metros).

Foram utilizados 14 ovinos, machos inteiros, mestiços Santa Inês, com peso vivo médio de 42,5 $( \pm 7,5) \mathrm{kg}$, distribuídos ao acaso em dois grupos experimentais com sete animais cada, denominados Grupo Controle e Grupo Infectado, e mantidos em sistema de confinamento total.

Os animais receberam silagem de sorgo ad libitum e suplemento concentrado constituído por farelo de soja, farelo de milho, farelo de trigo, premix de ovino (PRODUMIX ${ }^{\circledR}$ ) e calcário calcítico, apresentando a formulação $16 \%$ de proteína bruta $(\mathrm{PB})$ e $73,8 \%$ de nutrientes digestíveis totais $(\text { NDT })^{(9)}$ uma vez ao dia na quantidade de $200 \mathrm{~g}$ /animal. Além disso, receberam suplemento mineral Ovinofós ${ }^{\circledR}$ com minerais orgânicos (suplemento mineral pronto para uso da Tortuga ${ }^{\circledR}-$ Linha de nutrição para ovinos) ad libitum.

O período experimental teve duração de 85 dias, nos quais os primeiros 14 dias foram destinados à adaptação dos animais e à realização da pesagem, identificação e avaliação quanto à presença de parasitos da superfamília Strongyloidea por meio da contagem de ovos por grama de fezes (OPG) e separação dos animais em dois lotes com sete animais cada. Os animais positivos foram vermifugados com fosfato de levamisol, na dosagem de $1 \mathrm{~mL} / 50 \mathrm{~kg}$ de peso vivo. Sete dias após o tratamento, repetiu-se o exame e todos os animais apresentaram OPG negativo para ovos do tipo Strongyloidea.

Após o período de adaptação, um dos grupos de animais recebeu por via oral uma suspensão larval contendo 10.000 larvas $\left(\mathrm{L}_{3}\right)$ de $H$. contortus obtidas a partir da coprocultura ${ }^{(10)}$ de fezes coletadas diretamente da ampola retal de 10 animais positivos, sendo a elaboração da suspensão larval e inoculação de acordo com normas da World Association for the Advancement of Veterinary Parasitology (WAAVP) ${ }^{(11)}$.

Para o início das coletas de dados, foi respeitado o período pré-patente (PPP) de 21 dias após a inoculação (DPI). O intervalo de coleta de dados foi semanal, totalizando 70 dias de observação, com nove momentos de observação divididos da seguinte forma: M0 - dia da inoculação, M21 - 21 dias, M28 - 28 dias, M35 - 35 dias, M42 - 42 dias, M49 - 49 dias, M56 - 56 dias, M63 - 63 dias e M70 -70 dias.

Foram avaliados os seguintes parâmetros: OPG, Famacha ${ }^{\odot}$, peso, proteínas plasmáticas totais e hematócrito. As análises de OPG foram realizadas segundo a técnica de Gordon e Withlock ${ }^{(12)}$. Para determinação do peso, foram realizadas pesagens individuais em balança após jejum de 16 horas. As coletas de sangue foram realizadas por meio de venopunção jugular, utilizando-se agulhas e tubos vacutainers com anticoagulante EDTA $10 \%$, identificados com o número do animal. Imediatamente após as coletas, o sangue foi conduzido ao laboratório para determinação do hematócrito e dosagem das proteínas plasmáticas totais, segundo a técnica e os valores de referência ${ }^{(13)}$. A análise Famacha ${ }^{\odot}$ foi realizada por meio da exposição da conjuntiva dos animais e comparação com o cartão modelo, graduado em escala de 1 a 5 . Ao final do período experimental, os animais foram abatidos e o trato gastrintestinal coletado para contagem e identificação dos parasitos adultos. 
Os dados foram submetidos ao teste de distribuição normal Shapiro-Wilk e todos os dados apresentaram distribuição normal admitindo-se $\alpha=0,05$. O teste ANOVA foi aplicado em cada grupo experimental para se avaliar cada momento de observação, seguido pela análise pos hoc pelo teste de Tukey, admitindo-se $95 \%$ de significância $(\mathrm{p}<0,05)$. Para comparação das médias entre os grupos experimentais em cada momento de observação, foi empregado o teste $t$ de Student. O programa utilizado para realização das análises estatísticas foi o Bioestat $5.0^{\circledR}$ e as figuras desenvolvidas no software GraphPad Prism® 6 Demo.

\section{Resultados}

No grupo infectado experimentalmente com larvas de $H$. contortus, o OPG apresentou níveis elevados a partir do M0 ( $<<0,0001)$, atingindo o maior média aos 42 dias pós infecção (M42). Uma redução significativa do OPG foi observada em M49, M56, M63 e M70, conforme observado na Figura 1. O grupo controle se manteve negativo durante todo o período experimental, não apresentando, portanto, diferenças entre os momentos analisados.

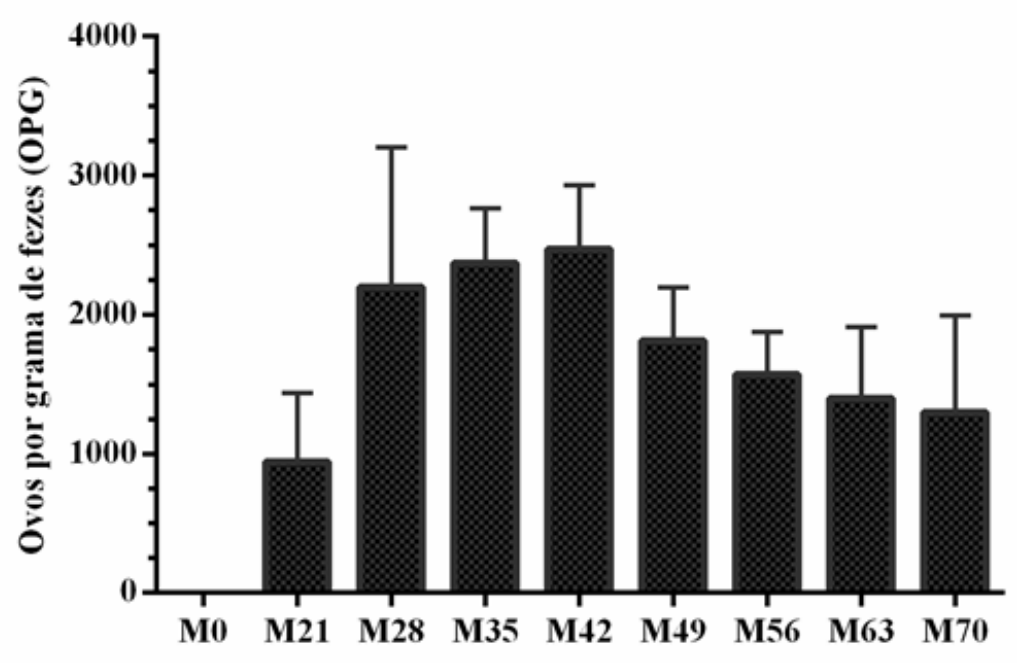

Figura 1. Médias e desvio padrão de ovos por grama de fezes (OPG) de ovinos mestiços Santa Inês infectados experimentalmente com larvas de $H$. contortus (grupo infectado) em nove tempos de observação (M0 - dia da inoculação, M21 - 21 dias, M28 - 28 dias, M35 35 dias, M42 - 42 dias, M49 - 49 dias, M56 - 56 dias, M63 - 63 dias e M70 - 70 dias). O OPG em M21, M28, M35, M42, M49, M56, M63 e M70 foi significativamente maior que M0 $(\mathrm{p}<0,0001)$. Teste ANOVA.

O hematócrito foi significativamente menor a partir da infecção experimental no grupo infectado $(\mathrm{p}=0,0025)$. A comparação entre o grupo infectado e o controle mostrou que, a partir de M0, em todos os momentos de observação o hematócrito dos animais pertencentes ao grupo infectado foi menor que do grupo controle (Figura 2). 


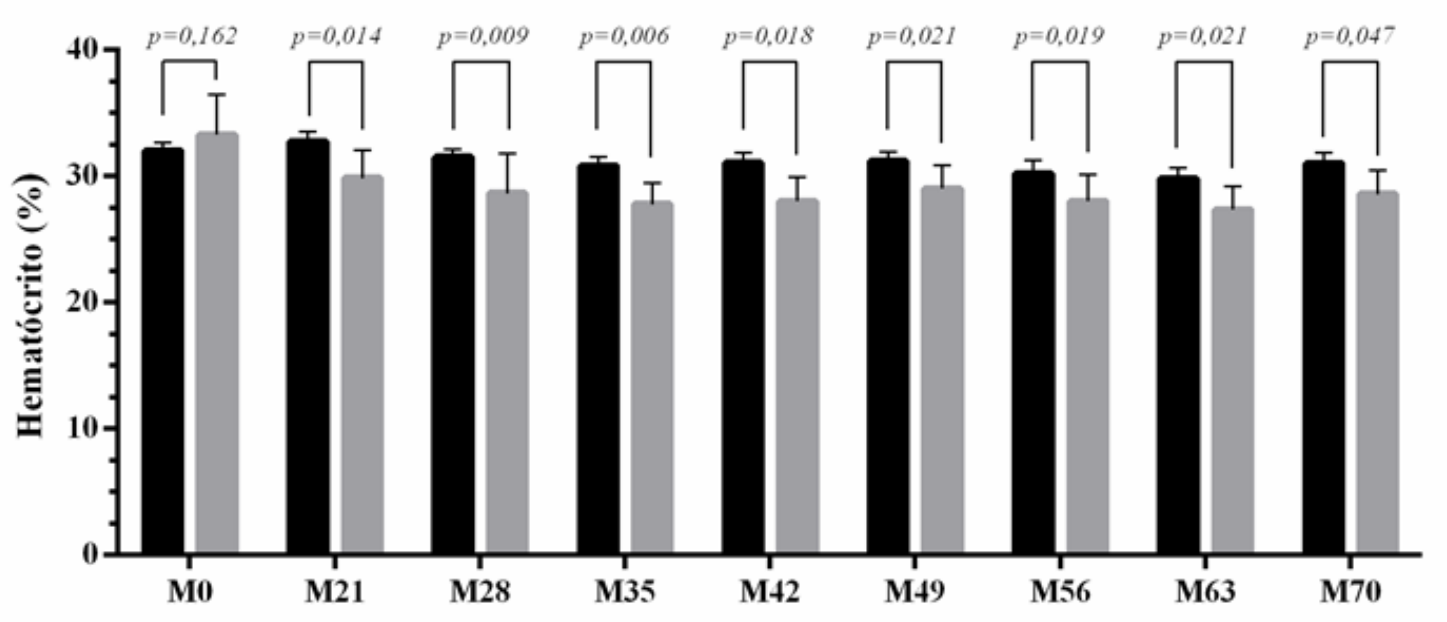

GRUPO CONTROLE

GRUPO INFECTADO

Figura 2. Médias e desvio padrão de hematócrito (\%) de ovinos mestiços Santa Inês infectados experimentalmente com larvas de $H$. contortus (grupo infectado) em comparação a ovinos livres de infecção parasitária (grupo controle), em nove tempos de observação (M0 dia da inoculação, M21 - 21 dias, M28 - 28 dias, M35 - 35 dias, M42 - 42 dias, M49 - 49 dias, M56 - 56 dias, M63 - 63 dias e M70 - 70 dias). Após M0, o hematócrito dos ovinos do grupo infectado foi significativamente menor que no grupo controle. Teste t de Student.

No grupo infectado, a análise Famacha ${ }^{\oplus}$ mostrou que o escore variou de 1 a 2 e foi significativamente maior em M21 (p=0,0103), M28 (p=0,0103), M35 (p=0,0023) e M42 ( $p=0,0023)$ em comparação ao escore observado no grupo controle.

Os resultados das análises empregadas para proteínas plasmáticas totais não apresentaram diferença significativa entre os momentos de observação, tanto para o grupo infectado $(\mathrm{p}=0,0917)$ quanto para o grupo controle $(\mathrm{p}=0,6493)$. As comparações dos valores de proteínas plasmáticas totais entre os grupos também não foram significativamente diferentes em todos os momentos de observação e se mantiveram dentro dos valores de referência para a espécie (6.0 a 7.9 g/dl) (Figura 3A). O parâmetro peso não apresentou diferença significativa dentro de cada grupo experimental, bem como entre os grupos controle e infectado (Figura 3B).

Após a necropsia dos animais, todos os helmintos foram coletados e identificados em laboratório como da espécie Haemonchus contortus. 
A

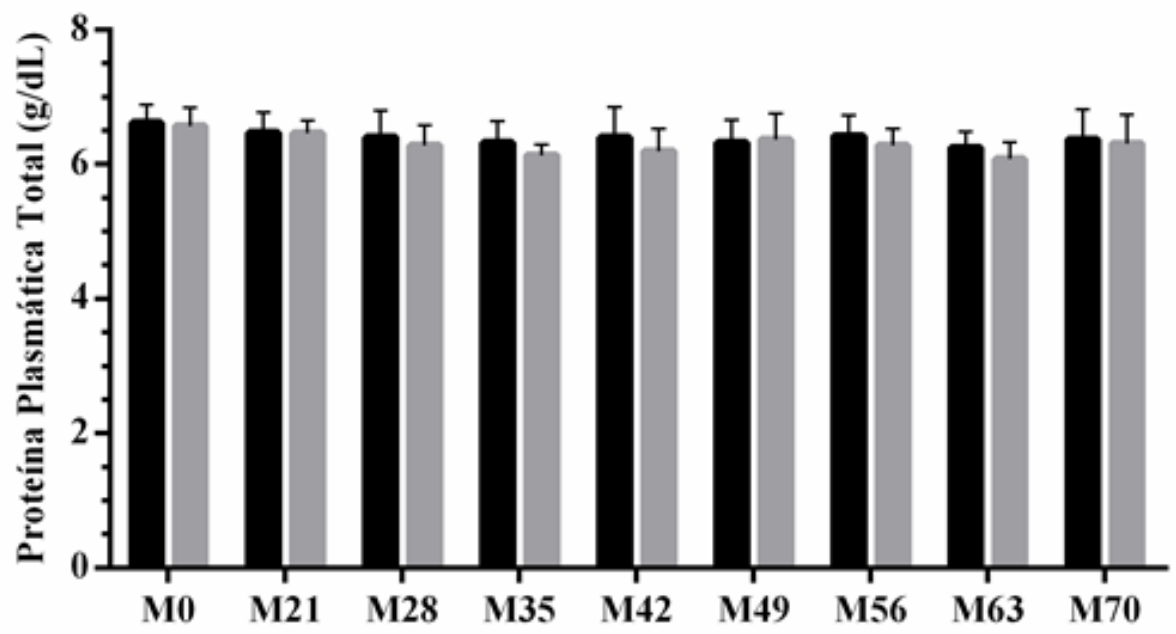

$\square$ GRUPO CONTROLE GRUPO INFECTADO

B

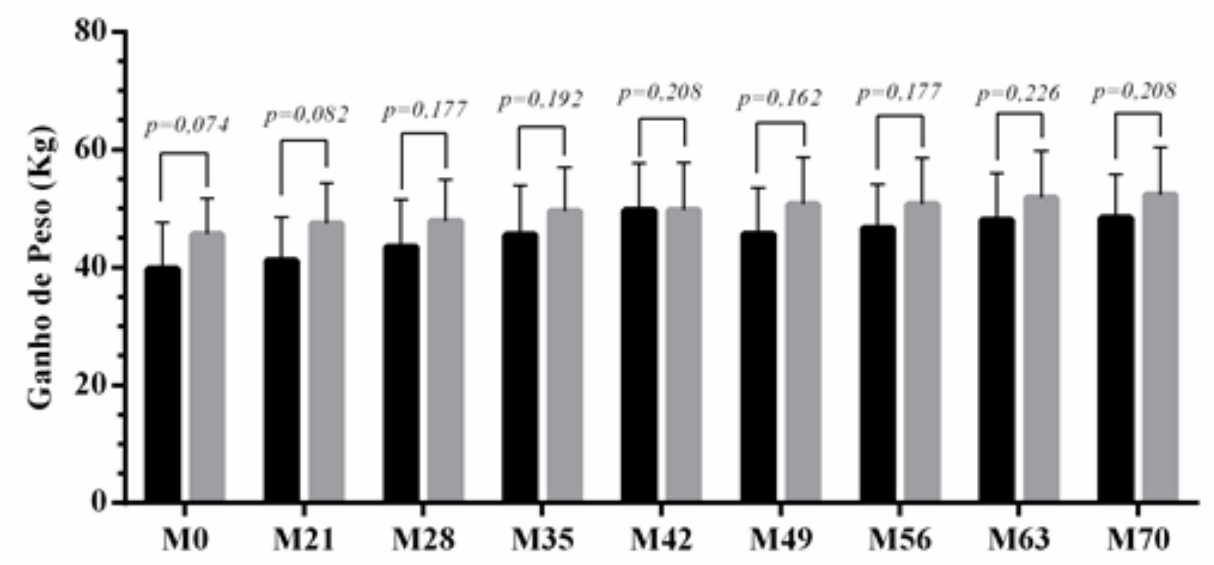

GRUPO CONTROLE

GRUPO INFECTADO

Figura 3 - Avaliação da proteína plasmática total (PPT) e peso de ovinos mestiços Santa Inês infectados experimentalmente com larvas de $H$. contortus (grupo infectado) em comparação a ovinos livres de infecção parasitária (grupo controle), em nove tempos de observação (M0 dia da inoculação, M21 - 21 dias, M28 - 28 dias, M35 - 35 dias, M42 - 42 dias, M49 - 49 dias, M56 - 56 dias, M63 - 63 dias e M70 - 70 dias). A - Comparação das médias e desvios padrão em $\mathrm{g} / \mathrm{dL}$ de $\mathrm{PPT}$ evidenciando que não houve alteração significativa dentro e entre os grupos. Teste ANOVA e Teste t Student. B - Comparação das médias e desvios padrão do peso mostrando não haver diferença significativa entre o grupo controle e o grupo infectado. Teste t Student. 


\section{Discussão}

Os resultados obtidos mostraram que a infecção experimental com 10.000 larvas de $H$. contortus causou alteração no hematócrito dos ovinos, porém, essa alteração não foi suficiente para comprometer clinicamente os animais, dadas as observações dos demais parâmetros como a proteína plasmática total, o método Famacha ${ }^{\odot}$ e o ganho de peso total. O nível de proteína plasmática total e peso corpóreo não foram afetados pela infecção.

Após o período pré-patente de 21 dias, o OPG nos animais infectados experimentalmente aumentou progressivamente até atingir seu maior valor aos 42 dias de observação, decaindo nas semanas seguintes até o final do experimento. A redução do OPG do grupo infectado a partir $42^{\circ}$ dia pode ser justificada pelo aumento de resistência dos animais, o que limita o estabelecimento de larvas infectantes, o crescimento e a fecundidade dos nematoides e pode até mesmo levar à eliminação dos parasitos presentes, como também foi descrito em trabalhos desenvolvidos por Bricarello et al. ${ }^{(4)} \mathrm{e}$ Amarante et al. ${ }^{(6)}$.

Em pequenos ruminantes, a intensidade da anemia e da hipoproteinemia são usualmente os indicadores da gravidade das parasitoses gastrintestinais, principalmente as ocasionadas pelo parasito H. contortus $^{(7)}$. Por isso, estudos que associam alterações hematológicas, como do volume globular ao OPG, podem auxiliar no diagnóstico e na seleção de animais resistentes ${ }^{(8)}$.

A menor média de hematócrito encontrada coincidiu com o momento em que a média do OPG esteve mais elevada. Uma forma de avaliar o grau de anemia é o método Famacha ${ }^{\odot}$. No Brasil, Molento et al. ${ }^{(14)}$ usaram o método Famacha ${ }^{\odot}$ como parâmetro clínico na identificação de ovinos e caprinos parasitados por $H$. contortus e comprovaram a aplicabilidade em ambas as espécies. Segundo Vatta et al. ${ }^{(15)}$, a eficiência do Famacha ${ }^{\odot}$ torna possível tratar somente os animais que sofrem de parasitismo severo, evitando o tratamento daqueles que não apresentam anemia clínica, assim, a pressão de seleção para a resistência aos anti-helmínticos torna-se menos intensa.

Apesar de o hematócrito e Famacha ${ }^{\odot}$ estarem significativamente alterados nos momentos de maior OPG, esses parâmetros se mantiveram dentro daqueles considerados de referência para a espécie durante todo o período experimental $(24 \text { a } 50 \%)^{(10)} \mathrm{e}$, neste caso, a classificação Famacha ${ }^{\odot}$ não sugere tratamento, uma vez que a vermifugação é indicada somente para animais com graus Famacha ${ }^{\circledR} 3,4$ e 5, ou seja, que apresentam sinais clínicos de anemia segundo a avaliação da conjuntiva, o que não ocorreu mesmo nos dias 28, 35, 42, 49 e 56, dias em que o OPG do grupo infectado foi superior a 1500. Esses resultados confirmam os encontrados em trabalho desenvolvido por Molento et al. ${ }^{(16)} \mathrm{e}$ Vieira et al. ${ }^{(17)}$, em que animais com OPG acima de 1500 não apresentaram clinicamente sinais de anemia.

Em trabalho desenvolvido por Chagas et al. ${ }^{(18)}$, foi observado controle satisfatório de endoparasitoses em ovinos tratando somente os animais com OPG igual ou superior a 4000. Esses resultados contradizem as informações de Ueno e Gonçalves ${ }^{(19)}$, que classificaram as infecções por nematódeos gastrintestinais como grau leve na faixa de 500 a 800 ovos, moderado de 800 a 1.500 ovos e elevado cima de 1.500 ovos, neste último é indicada a intervenção com o uso de anti-helmíntico. Assim, baseados nos resultados de OPG em associação ao hematócrito e método Famacha $^{\circledR}$, nossos 
resultados ressaltam a necessidade de avaliação conjunta de diversos parâmetros nas indicações de intervenções anti-helmínticas, não considerando apenas o OPG.

Não houve diferença significativa nos níveis de proteína plasmática total, tanto nos diferentes momentos de observação, como entre os grupos experimentais, estando os mesmos dentro dos valores de referência para a espécie. Esses resultados corroboram os encontrados por Kawano et al. ${ }^{(20)}$, Bricarello et al. ${ }^{(4)}$ e Souza et al. ${ }^{(21)}$, nos quais ovinos parasitados também não apresentaram alterações nas concentrações de proteínas plasmáticas totais.

Os fatores que mais causam prejuízos relacionados à verminose são o retardo do crescimento, perda de peso, baixa fertilidade e, em casos mais graves, altas taxas de mortalidade ${ }^{(1)}$. Na condição experimental, não houve diferença significativa entre o peso de animais infectados e aqueles não infectados. Esse fato pode ser atribuído ao suplemento na alimentação dos animais e ao seu confinamento, o que manteve a condição imunológica dos animais e diminuiu o risco de reinfecções. Em indivíduos nos quais a resposta imunológica contra os parasitos adultos é eficaz, observa-se redução no crescimento, na fecundidade, mudanças morfológicas e a expulsão da população de nematoides pelo hospedeiro, sendo esta mais eficiente em função da imunidade adquirida, em consequência de repetidas infecções do hospedeiro ao longo de sua vida ${ }^{(22)}$.

O estado de saúde geral dos animais e a equivalência dos diversos parâmetros analisados nos grupos experimentais podem ser explicados tendo como embasamento fatores intrínsecos ou regulados geneticamente e fatores ambientais. Entre os fatores intrínsecos, a resposta à infecção, que limita ou impede o estabelecimento de alterações sistêmicas no hospedeiro provocadas pelo parasitismo gastrintestinal, está ligada à resistência ou à tolerância, sendo a resistência a capacidade do hospedeiro em desenvolver uma resposta imune que limita o estabelecimento do parasito e a tolerância a capacidade dos animais de conviver com os parasitos apresentando redução mínima na produtividade $^{(23)}$.

Até o $42^{\circ}$ dia, os animais apresentaram um aumento progressivo nos valores de OPG, sem observação clínica ou laboratorial de sinais de anemia e alterações nas concentrações de proteínas plasmáticas totais, o que caracteriza a tolerância dos animais frente à infecção. Ainda, a partir do $42^{\circ}$ dia, os valores de OPG foram diminuindo progressivamente até o último dia de análise e os animais continuaram sem alterações clínicas e laboratoriais, o que sugere a resistência desenvolvida pelos animais frente à infecção, já limitando o estabelecimento dos parasitos. Em trabalhos desenvolvidos por diversos autores, ovinos da raça Santa Inês se destacam em resistência quando comparados aos animais das raças Suffolk, Ile de France e Poll Dorset ${ }^{(24-27)}$.

Dessa forma, a rusticidade e a boa adaptação desses animais ao ambiente podem ser fatores associados aos resultados encontrados. Entretanto, os fatores ambientais dependem principalmente da qualidade da dieta fornecida aos animais. Assim, se a dieta atender às exigências nutricionais, especialmente no que se refere a níveis de proteína, os animais conseguirão suportar mais facilmente as infecções parasitárias ${ }^{(26,28)}$. De acordo com Silva et al. ${ }^{(29)}$, o uso de dietas ricas em proteína pode contribuir com a resposta imunológica gerada pelos animais em resposta à infecção parasitária, proporcionando desempenho satisfatório de raças susceptíveis, apesar de albergarem os parasitos. Com medidas de seleção e manejo, reduz-se a necessidade de intervenção anti-helmíntica e, consequentemente, o desenvolvimento de resistência parasitária aos fármacos disponíveis no mercado. 


\section{Conclusão}

A infecção experimental com 10.000 larvas de $H$. contortus e OPG até 2500 não afetam significativamente o peso, o hematócrito e a proteína plasmática total de ovinos mestiços Santa Inês confinados, não havendo necessidade de intervenção anti-helmíntica.

\section{Referências}

1. Vieira LS. Métodos alternativos de controle de nematoides gastrintestinais em caprinos e ovinos. Rev. Tecnol. Cien. Agropec. 2008; 2(2): 28-31.

2. Macedo FAF, Siqueira ER, MARTINS EL. Análise econômica da produção de carne de cordeiros sob dois sistemas de terminação: pastagem e confinamento. Cienc. Rural 2000; 30(4): 677-680.

3. Saddiqi HA, Jabbar A, Sarwar M, Iqbal Z, Muhammad G, Nisa M, et al. Small ruminant resistance against gastrointestinal nematodes: a case of Haemonchus contortus. Parasitol. Res. 2011; 109(2): 1483-1500.

4. Bricarello PA, Amarante AFT, Rocha RA, Cabral Filho SL, Huntley JF, Houdijk JGM, et al. Influence of dietary protein supply on resistance to experimental infections with Haemonchus contortus in Ile de France an Santa Ines lambs. Vet. Parasitol. 2005; 134(2): 99-109.

5. Vieira, LS. Endoparasitoses gastrintestinais em caprinos e ovinos. Sobral: Embrapa Caprinos. Embrapa Caprinos. Documentos, 2005.

6. Amarante AFT, Rocha RA, Bricarello PA. Relationship of intestinal histology with the resistance to Trichostrongylus colubriformis infection in three breeds of sheep. Pesq. Vet. Bras. 2007 Jan; 27(1): 43-48.

7. Chakraborty D, Lodh C. Blood biochemical profiles in Fasciola, Haemonchus and Diectyocaulus species infectin in goat - A comparative study. Infian. Vet. J. 1994; 71(3): 286-288.

8. Gauly M, Erhardt G. Genetic resistance to gastrointestinal nematode parasites in Rhön sheep following natural infection. Vet. Parasitol. 2001; 102(3): 253-259.

9. NRC. National Research Council. Nutrient requirements of small ruminants. Washington: National Academic Press; 2007.

10. Roberts FHS, O'sullivan JP. Methods for egg count sand larval cultures for strongyles infesting the gastrointestinal tract of cattle. Aut. J. Agric. Res. 1950; 1(1): 99-102.

11. World Association for the Advancement of Veterinary Parasitology (W.A.A.V.P.) second edition of guidelines for evaluating the efficacy of anthelmintics in ruminants (bovine, ovine, caprine). Vet. Parasitol. 1995; 58(1): 181-213.

12. Gordon HMC, Whitlock HV. A new technique for counting nematode eggs in sheep faeces. Journal of Commonwealth Science and Industry Research Organization 1939; 12(1): 50-52.

13. Jain NC. Essentials of Veterinary Hematology. Philadelphia: Lea \& Febiger, 1993, Cap. 2. 470p.

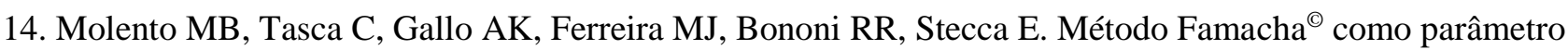
clínico individual de infecção por Haemonchus contortus em pequenos ruminantes. Cienc. Rural 2004; 34: 1139- 1145 . 
15. Vatta AF, Letty BA, Van der Linde MJ, Van Wijk EF, Hansen JW, Krecek RC. Testing for clinical anaemia caused by Haemonchus spp. in goats farmed under resource-poor conditions in South Africa using an eye colour chart developed for sheep. Vet. Parasitol. 2001; 99: 1-14.

16. Molento MB. Resistência de helmintos em ovinos e caprinos. In: CONGRESSO BRASILEIRO DE PARASITOLOGIA VETERINÁRIA \& I SIMPÓSIO LATINO-AMERICANO DE RICKETISIOSES, 13., 2004, Ouro Preto, MG. Rev. Bras. Parasitol. Vet. 2004; 13.

17. Vieira MIB, Rocha HC, Ractz LAB, Moraes RB, Oliveira IS. Variação anual de peso vivo e ovos por grama de fezes de borregas e ovelhas submetidas a dois métodos de controle do Haemonchus contortus. Arch. Vet. Sci. 2007; 12(2): 5-6.

18. Chagas ACS, Oliveira MCS, Fernandes LB, Machado R, Esteves SN, Sales RL, et al. Ovinocultura: controle da verminose, mineralização, reprodução e cruzamentos na Embrapa Pecuária Sudeste. Documentos, 65. Embrapa Pecuária Sudeste, São Carlos, SP, 2008.

19. Ueno H, Gonçalves PC. Manual para diagnóstico das helmintoses de ruminantes. Tokyo: Japan International Cooperation Agency; 1998.

20. Kawano EL, Yamamura MH, Ribeiro ELA. Efeitos do tratamento com anti-helmíntico em cordeiros naturalmente infectados com helmintos gastrintestinais sobre os parâmetros hematológicos, ganho de peso e qualidade da carcaça. Arq. Facul. Vet. RS. 2001; 29(2): 113-121.

21. Souza C, Lopes STA, Batina PN, Cecim M, Cunha CM, Conrado AC, et al. Estresse parasitário em cabras Saanen: Avaliação hematológica e da atividade oxidativa dos neutrófilos. Vet. Not. 2006; 12(2): 17-23.

22. Balic A, Bowles VM, Meeusen ENT. The Immunobiology of gastrointestinal nematode infections in ruminants. Adv. Parasitol. 2000; 45: 181-241.

23. Alberts GAA, Gray GD, Piper LR. The genetic resistance and resiliense to Haemonchus contortus infection in young Merino sheep. Int. J. Parasitol. 1987; 17: 1355-1363.

24. Moraes FR, Thomaz-Soccol V, Rossi Junior P. Susceptibilidade de ovinos das raças Suffolk e Santa Inês à infecção natural por tricostrongilídeos. Arch. Vet. Sci. 2000; 6: 63-69.

25. Bueno MS, Cunha EA, Verissimo CJ. Infección por nematodos em razas de ovejas carniças criadas intensivamente em la región del Sudeste del Brasil. Arch. Zoot. 2002; 51: 273-280.

26. Bricarello PA, Amarante AFT, Houdijk JGM. Influence supply on resistance to infection with Haemonchus contortus in Ile de France and Santa Inês. In: THE BRITISH SOCIETY OF ANIMAL SCIENCE, 2003, York. Proceedings... York: BASA, p. 93, 2003.

27. Amarante AFT, Bricarello PA, Rocha RA, Genari SM. Resistance of Santa Ines, Suffolk and Ile de France lambs to naturally acquired gastrointestinal nematode infections. Vet. Parasitol. 2004; 120: 91-106.

28. Knox MR., Steel JW. The effects of urea supplementation on production and parasitological responses of sheep infected with Haemonchus contortus and Trichostongylus colubriformis. Vet. Parasitol. 1999; 83: 123135.

29. Silva BF, Bassetto CC, Shawb RJ, Canavessic AMO, Amarante, AFT. Parasitism by Oestrus ovis: Influence of sheep breed and nematode infections. Vet. Parasitol. 2012; 186: 437-444. 